\title{
$\alpha$-Smooth muscle actin expression and desmoplastic stromal reaction in pancreatic cancer: results from the CONKO-001 study
}

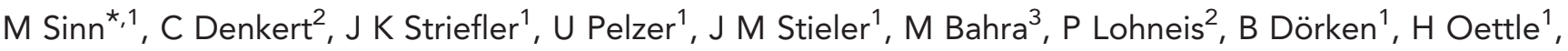 \\ $\mathrm{H}$ Riess $^{1}$ and B V Sinn ${ }^{2}$ \\ ${ }^{1}$ Charité-Universitätsmedizin Berlin, Department of Medical Oncology and Haematology, Augustenburger Platz 1, 13353 Berlin, \\ Germany; ${ }^{2}$ Charité-Universitätsmedizin Berlin, Institute of Pathology, Chariteplatz 1, 10117 Berlin, Germany and ${ }^{3}$ Charité- \\ Universitätsmedizin Berlin, Department of General, Visceral and Transplantation Surgery, Augustenburger Platz 1, 13353 Berlin, \\ Germany
}

Background: Previous investigations in pancreatic cancer suggest a prognostic role for $\alpha$-smooth muscle actin $(\alpha$-SMA) expression and stromal density in the peritumoural stroma. The aim of this study was to further validate the impact of $\alpha$-SMA expression and stromal density in resectable pancreatic cancer patients treated with adjuvant gemcitabine compared with untreated patients.

Methods: CONKO-001 was a prospective randomised phase III study investigating the role of adjuvant gemcitabine as compared with observation. Tissue samples of 162 patients were available for immunohistochemistry on tissue microarrays to evaluate the impact of $\alpha$-SMA expression and stromal density impact on patient outcome.

Results: High $\alpha$-SMA expression in tumour stroma was associated with worse patient outcome (DFS: $P=0.05, \mathrm{OS}: P=0.047)$. A dense stroma reaction was associated with improved disease-free survival (DFS) and overall survival (OS) in the overall study population (DFS: $P=0.001, \mathrm{OS}: P=0.001$ ). This positive prognostic impact was restricted to patients with no adjuvant treatment (DFS: $P<0.001$, OS: $P<0.001)$. In multivariable analysis, $\alpha$-SMA and stromal density expression were independently predictive factors for survival.

Conclusions: Our data confirm the negative prognostic impact of high $\alpha$-SMA expression in pancreatic cancer patients after curatively intended resection. In contrast to former investigations, we found a positive prognostic impact for a dense stroma. This significant influence was restricted to patients who received no adjuvant therapy.

The abundant desmoplastic stroma reaction in pancreatic cancer is considered to be an important reason for its aggressive tumour biology and resistance to chemotherapy (Feig et al, 2012). The depletion of the peritumoural stroma seems to be a promising therapeutic point of action (Hamada et al, 2013) and targeting the interactions between the so called tumour microenvironment-especially pancreatic stellate cells-and tumour cells may be an answer for the consistently sobering therapeutic resistance of the disease. This conclusive theory is complicated by the fact that the tumour microenvironment must be understood as a complex and not static entity including pancreatic stellate cells, fibroblasts, myofibroblasts, immune cells, blood vessels, extracellular matrix and soluble proteins such as cytokines and growth factors (Erkan et al, 2012a, b; Lunardi et al, 2014). In this context, the role of $\alpha$-smooth muscle actin $(\alpha$-SMA) that is expressed by activated pancreatic stellate cellsand maybe a marker for stroma activation-seems to be of special interest and former results suggest a prognostic relevance of $\alpha$-SMA for resected pancreatic cancer patients (Erkan et al, 2008; Fujita et al, 2010). 
The recent approval of the albumin-bound taxane nabpaclitaxel in the combination with the standard therapeutic agent gemcitabine as palliative first-line therapy in the United States and subsequently in Europe based on the results of the MPACT trial (Von Hoff et al, 2013) directs the interest of medical oncologists even more in this direction because of the fact that one effect of nab-paclitaxel seems to be a direct attack against the peritumoural stroma (Von Hoff et al, 2011). We have recently described the predictive role of SPARC as an important marker of the peritumoural stroma reaction in resected pancreatic cancer patients treated with gemcitabine (Sinn et al, 2014).

Going a step further and considering the degree of stroma reaction as a potential and clinically relevant prognostic and eventually even predictive biomarker, we are faced to the fact that no standardised method for the evaluation of this important marker exists so far. For decades, surgeons linked malignant pancreatic tumours to 'rocks' (Garber, 2010), but no clear descriptive or quantitative 'stroma classification' can be found beyond that. Neither the quantification nor the evaluation of the quality of 'peritumoural stroma' is part of the clinical routine.

As a prospective phase III trial, the CONKO-001 investigated the efficacy of adjuvant gemcitabine and established adjuvant treatment as the standard of care in R0- or R1-resected pancreatic cancer patients (Oettle et al, 2007; Seufferlein et al, 2013). The study can provide prospectively collected data and a follow-up of more than 5 years, thereby being an optimal starting point for translational research in pancreatic cancer.

The objective of our here-presented study was to analyze $\alpha$-SMA that is produced by activated pancreatic stellate cells (Wilson et al, 2014) as a marker of stroma quality and to correlate these findings with disease-free survival (DFS) and overall survival (OS). We supposed to find high $\alpha$-SMA expression as a negative prognostic factor in resected pancreatic adenocarcinoma. In a second step, we investigated the peritumoural stroma by a standardised, but qualitative-in terms of descriptive-method. We acted on the assumption that dense stromal reaction would be correlated with a worse prognosis in terms of decreased DFS and OS.

\section{METHODS}

CONKO-001: baseline data. CONKO-001, a prospective randomised phase III study, investigated the role of adjuvant gemcitabine as compared with observation. A total of 368 patients with completely resected pancreatic cancer (R0 and $\mathrm{R} 1$ resection) were recruited between July 1998 and December 2004. Treatment with gemcitabine $\left(1000 \mathrm{mg} \mathrm{m}^{-2} \mathrm{~d} 1,8,15, \mathrm{q} 29\right)$ was continued for 6 months in an outpatient setting; subsequent follow-ups were at 8 -weekly intervals. Prior to the here-presented analysis, histological verification of adenocarcinoma was carried out by the local pathologist. Median DFS as the primary endpoint of the study was significantly improved by more than 6 months compared with observation only (13.4 vs 6.9 months, $P<0.001$ ) (Oettle et al, 2007). Overall survival after a prolonged follow-up demonstrated significant improvement too (22.8 vs 20.2 months, $P=0.005$ ) (Oettle et al, 2013).

The study was approved by the institutional review committee (trial registration isrctn.org Identifier: ISRCTN34802808).

Smooth muscle actin ( $\alpha$-SMA) and stroma evaluation. A total of 183 formalin-fixed paraffin-embedded tissue samples could be collected retrospectively. Evaluation of stromal density and $\alpha$-SMA expression was carried out on tissue microarrays in 162 patients containing three representative 1-mm tissue cores for each case. One hundred and sixty tumour samples were evaluable for $\alpha$-SMA and stromal density, respectively.

Immunohistochemistry for $\alpha$-SMA was performed according to standard procedures (DAKO, Hamburg, Germany; clone M0874, $1: 50)$. The slides were digitalised with their corresponding H\&Estained slides (Mirax Scan, Zeiss, Jena, Germany) and evaluated by virtual microscopy using the VMScope Silde Explorer (VMScope, Berlin, Germany) by two observers blinded to clinical outcome (MS, BVS). The H\&E-stained TMAs were interpreted in parallel to ensure smooth-muscle fibres of the duodenal wall were not mistaken for stromal reaction. The staining intensity was defined as negative, weak, moderate or strong. Negative and weak staining was subsumed to 'low $\alpha$-SMA' and moderate and strong to 'high $\alpha$-SMA'. Figure 1 shows weak $(1 \mathrm{~A}, \mathrm{C})$ and strong staining intensity (1B). The cutoff point for $\alpha$-SMA expression was chosen on the basis of the data distribution alone to avoid considerable differences of sample sizes without prior knowledge of clinical outcome.

The stromal density was evaluated on H\&E-stained TMAs. Quality was defined as loose, moderate or strong on the basis of its morphologic appearance. Loose stroma had a paucicellular matrix of loosely packed connective tissue fibres with occasional oedematous appearance. The stroma was defined as moderately dense when the network of connective fibres was denser but still with well-distinguishable fibres of connective tissue. Cases with dense stroma showed a densely packed network of fibres with intense staining. Figure 2 shows a loose (2A), moderate (2B) and dense (2C) peritumoural stroma reaction.

To reduce effects that might be caused by intratumoural heterogeneity, three different tumour areas were selected for the construction of TMAs. When different staining intensities were observed between the spots representing one case, an average was defined and used for analysis. To reduce effects caused by intraobserver variability, TMAs were evaluated by two observers. Ambiguous cases were discussed to gain agreement.

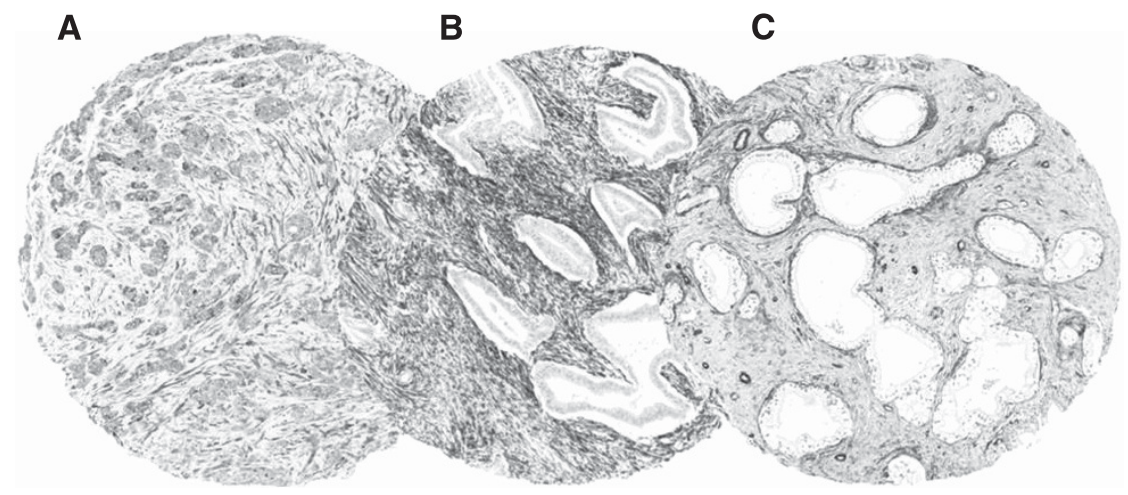

Figure 1. Expression of smooth-muscle actin in desmoplastic stroma. The figure illustrates examples with weak (A, C) and strong (B) staining intensity. 


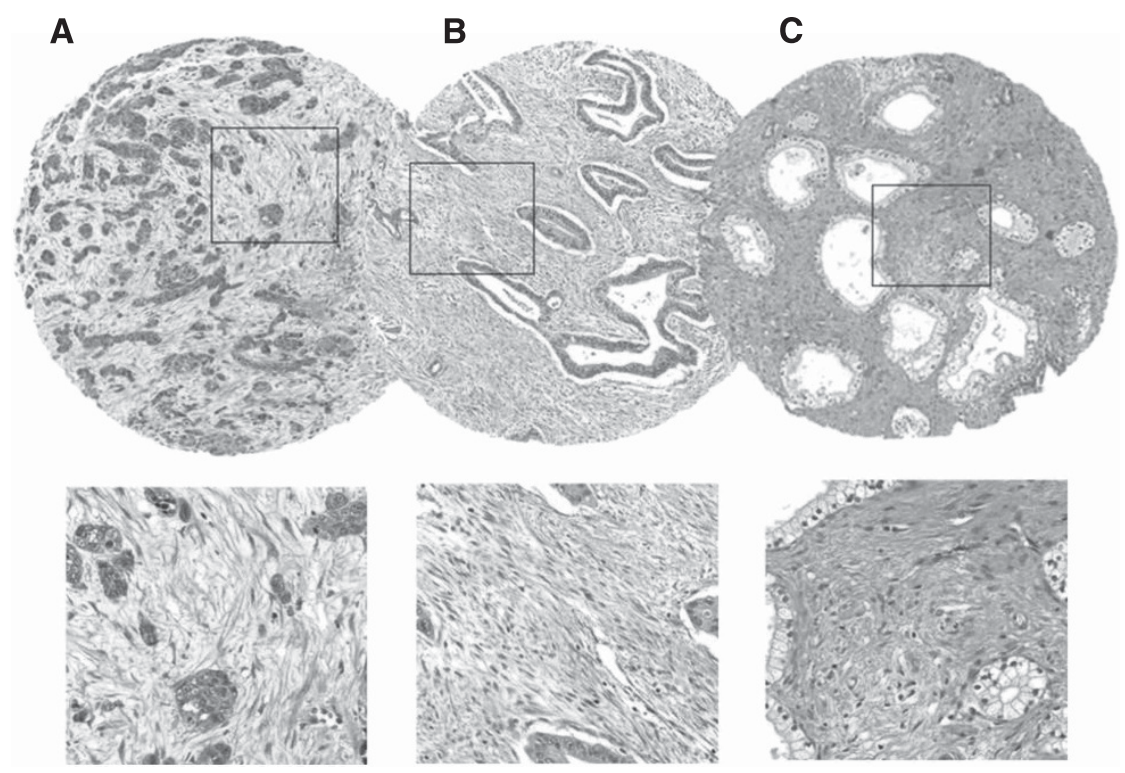

Figure 2. Patterns of density of desmoplastic stroma. (A) Loose connective tissue with myxoid appearance. (B) Moderate density. (C) Tightly packed connective tissue fibres (same cases as in Figure 1).

Statistical considerations. Disease-free survival (DFS) was defined as time from study entry to local or distant disease relapse, OS as time from study entry to death of any cause. The relation of stroma quality or $\alpha$-SMA expression with clinical and pathological tumour characteristics was evaluated using $\chi^{2}$ tests. The Kaplan-Meier method with log-rank tests was used for univariable survival analyses. The Cox model (Cox regression 1970) was used for multivariable analysis initially including all significant parameters and implementing a backward selection procedure with a cutoff value of $P<0.1$. In general, $P$ values were calculated two-sided and considered as significant when $<0.05$.

\section{RESULTS}

Patients' characteristics. The clinicopathological parameters for this translational investigation $(n=162)$ are shown in Table 1.

Stromal density and expression of $\alpha$-SMA. The intensity of stromal $\alpha$-SMA expression was weak in $25(16 \%)$, moderate in 87 (54\%) and strong in $46(29 \%)$ of 160 evaluable cases; 2 cases (1\%) were negative (Figure 1). Cases with negative and weak $\alpha$-SMA expression were summarised to a 'low' $(n=27,17 \%)$ and moderate and strong cases to a 'high' $(n=133,83 \%) \alpha$-SMA expression group.

The quality of stroma was defined as loose in 45 (28\%), moderate in $98(61 \%)$ and dense in $17(11 \%)$ of 160 evaluable cases (Figure 2).

There was a significant association between $\alpha$-SMA and stromal density $(P=0.005)$.

Correlation of $\alpha$-SMA expression/stromal density with clinical/ pathological data. Strong stromal $\alpha$-SMA had no significant association with tumour grading, $\mathrm{pT}$ stage, $\mathrm{pN}$ stage or resection margin R0/R1. Similarly, there was no significant association between stromal density and tumour differentiation (grading), tumour size (pT stage), presence of lymph node metastasis ( $\mathrm{pN}$ stage) or resection margin (R0/R1).

$\alpha$-SMA expression and impact on patient outcome. Negative/ weak $\alpha$-SMA expression was associated with longer DFS and OS (Figure 3): median DFS (OS) $\alpha$-SMA negative/weak $v s$ moderate/ strong 13.8 (28.0) vs 10.1 (20.2) months, $P=0.05$ (0.047).
This effect was not significant in the gemcitabine-treated group, where the median DFS (OS) comparing $\alpha$-SMA low $v s$ high was 15.1 (28.0) vs 12.6 (21.5) months, $P=0.073$ (0.061). In the observation group, $\alpha$-SMA expression had no significant influence on survival DFS (OS) as well and comparing $\alpha$-SMA low $v s$ high was 9.5 (20.2) months vs 6.2 (19.1) months; $P=0.282$ (0.323).

Stromal density and impact on patient outcome. In the overall study group, dense stroma was associated with a significant longer DFS $(P=0.001)$ and OS $(P=0.001)$ as compared with moderate or loose stroma (Figure 4). Median DFS (OS) for loose $v s$ moderate $v s$ dense stroma was 12.8 (28.0) vs 8.5 (18.5) vs 29.0 (46.0) months, respectively.

The significant effect of stromal density was restricted to patients of the observation group where the median DFS (OS) loose vs moderate vs dense was 10.1 (20.8) vs 5.8 (16.6) vs 42.0 (median not reached) months $(P<0.001)$. In patients who were treated with adjuvant gemcitabine, stromal density showed no significant effect with a median DFS (OS) loose vs moderate $v s$ dense with 17.8 (28.8) vs 12.3 (21.0) vs 14.9 (41.1) months; $P=0.204$ (0.093).

Interaction between treatment arm and stromal density was not significant $(P=0.083)$.

Univariable and multivariable analysis. $\alpha$-Smooth muscle actin expression and stromal density as well as tumour grading were significant factors in univariable analysis in regard to median DFS and OS. Treatment arm was significant for median DFS as well.

In a consecutive exploratory multivariable survival analysis for median DFS and OS, $\alpha$-SMA expression, stromal density, pT stage and tumour grading had a significant impact on patient survival (median DFS and OS), treatment arm on DFS (Table 2).

Impact of $\alpha$-SMA expression and stromal density on long-term survival (LTS). Patients with LTS were defined with an OS of 60 months or more. In a univariate analysis, low $\alpha$-SMA expression and dense stroma quality were significantly associated with LTS $(\alpha$-SMA expression and LTS, $P=0.044$; stroma quality and LTS, $P=0.010)$.

Correlation of $\alpha$-SMA expression and stromal density and stromal SPARC expression. The evaluation of stromal SPARC expression in the here-presented CONKO-001 subgroup was 
Table 1. Clinical and pathological patient characteristics of the study group and association with stromal density and $\alpha$-SMA expression

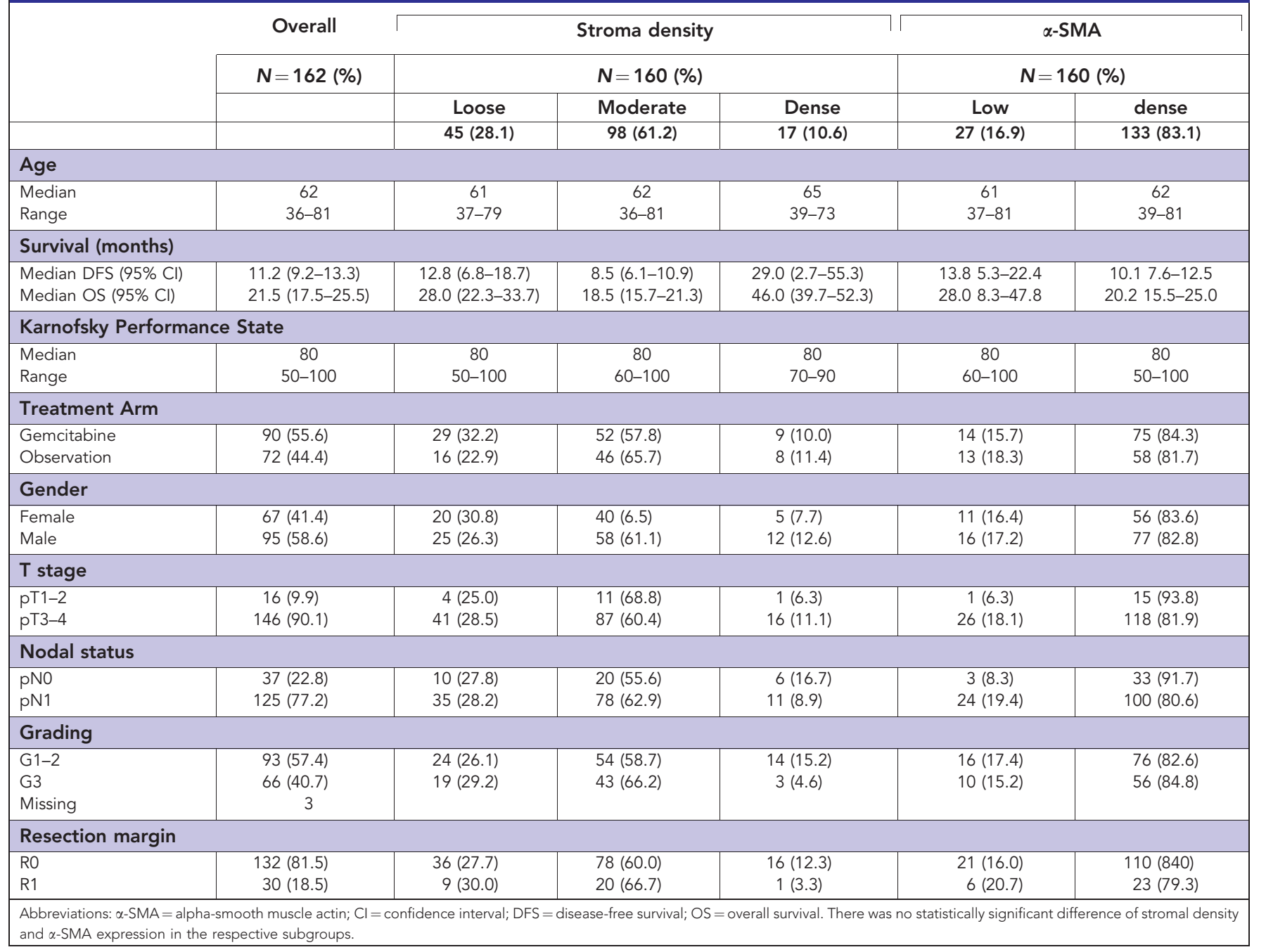
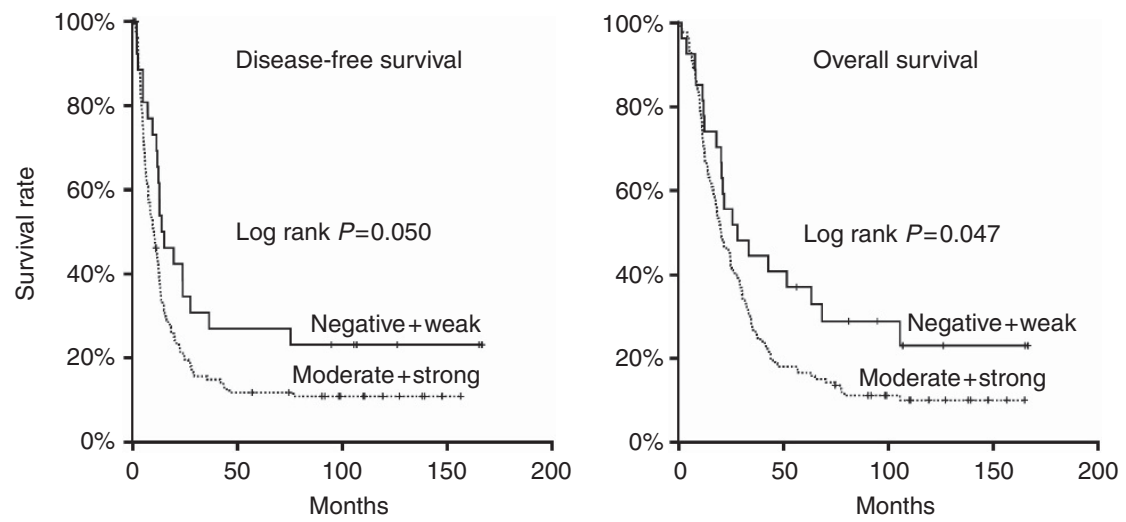

Figure 3. Kaplan-Meier curves: $\alpha$-SMA expression. High $\alpha$-SMA expression is associated with decreased DFS and OS in the overall study population.

described previously: a classification into two subgroups 'strong' and 'not strong' was carried out (Sinn et al, 2014).

Strong $\alpha$-SMA expression was associated with a strong stromal SPARC expression. This effect was only significant if the four subgroups of $\alpha$-SMA expression (negative, weak, moderate, strong) were compared with the two subgroups of stromal SPARC expression $(P=0.032)$, but not for the comparison low/high $\alpha$-SMA and strong/not strong SPARC.

There was no significant association between stromal SPARC expression and stromal density. 

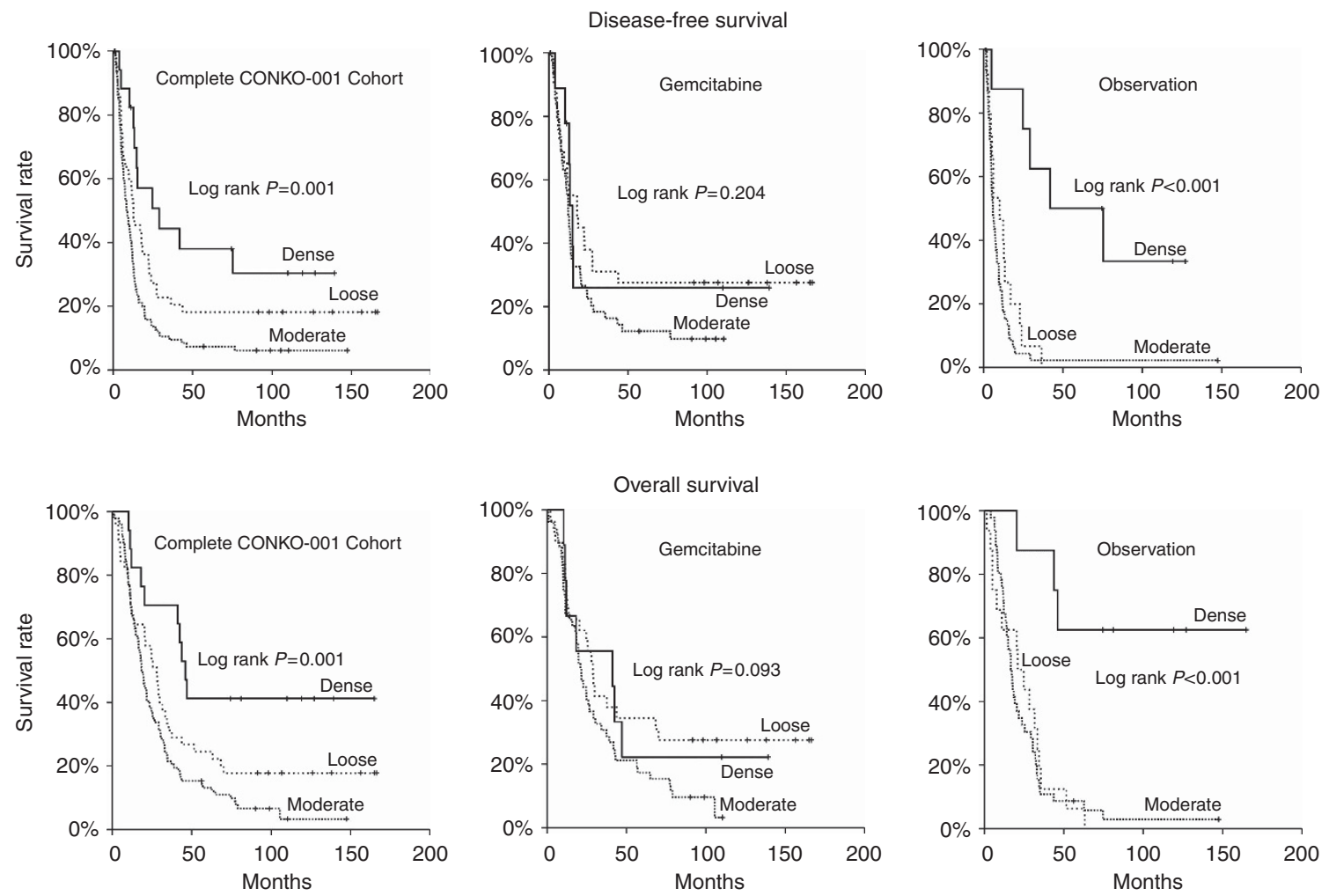

Figure 4. Kaplan-Meier curves: stromal density. A dense stroma is a positive prognostic biomarker for DFS and OS in patients that received no adjuvant treatment but not in patients who received adjuvant treatment with gemcitabine. Stroma density as well as DFS and OS are indicated by the different rows, treatment arms by the different columns.

\section{DISCUSSION}

To our best knowledge, this is the first evaluation of $\alpha$-SMA expression and stromal density in a large randomised phase III trial including patients with pancreatic adenocarcinoma after curatively intended surgery with or without adjuvant treatment.

In our here-presented study, we show that $\alpha$-SMA expression and stromal density are prognostic markers in resected pancreatic cancer patients. High $\alpha$-SMA expression was associated with a significant poorer DFS and OS in the overall study population. The positive effect for a dense stroma quality was highly significant in the observation group $(P=0.001)$, but not in patients who received adjuvant chemotherapy with gemcitabine.

Concerning $\alpha$-SMA expression, our results are in line with one study on 112 patients that described high $\alpha$-SMA mRNA levels as a negative prognostic marker in resected pancreatic cancer and correlated these results with immunohistochemical analysis (Fujita et al, 2010). In addition, in vitro cell cultures demonstrated that activated pancreatic stellate cells-producing $\alpha$-SMA-enhanced proliferation and colony formation of pancreatic cancer cells. In a preclinical mouse model, $\alpha$-SMA expression in pancreatic cancer tissue was significantly reduced after treatment with gemcitabine (Yamao et al, 2013).

$\alpha$-SMA is produced by activated pancreatic stellate cells and these fibroblast-like cells have multiple modulatory functions in the development of pancreatic cancer: activated by pancreatic cancer cells and activating these vice versa, they support the abundant stroma production present in most pancreatic cancers and promote tumour growth as well as invasiveness (Erkan et al, 2012a,b). They are related to be 'partner in crimes' with pancreatic cancer cells (Vonlaufen et al, 2008) and to target them-especially in combination with other stroma-depleting or immune-modulatory agents-seems to be one of the most interesting therapeutic approaches of the last decade (Ramírez et al, 2014). In this context, an interim analysis of a phase II study investigating the combination therapy of gemcitabine, nab-paclitaxel and the hedgehog inhibitor vismodegib showed promising results (DeJesus-Acosta et al, 2014).

Erkan et al (2008) tried to establish an 'activated stroma index' by a semi-automated computer-aided colour analysis evaluating $\alpha$-SMA expression and collagen staining, but this method has not become part of clinical daily care practise so far. In this investigation including 233 tumour specimens of resected pancreatic cancer patients, a high collagen deposition was demonstrated to be related with a significant improved survival.

This finding is in line with our results that demonstrated furthermore that not only collagen deposition but also a dense stroma quality in general may be a positive prognostic factor in pancreatic cancer. These results suggest furthermore that the role of pancreatic stroma is complex and depends on the composition and the density.

In this context, the matricellular protein SPARC (secreted protein acidic and rich in cysteine) is the best investigated biomarker so far and it seems to play a key role in the development of the dense collagenous stroma associated with pancreatic cancer lesions (Delany, 2010). Clinically, SPARC is of particular interest in pancreatic and other cancers, as it is might be a predictive marker for response to the albumin-bound taxan nab-paclitaxel or for chemotherapy in general (Sinn et al, 2014) and one effect of nabpaclitaxel seems to be stroma depletion (Von Hoff et al, 2011). In line with these theoretical approaches, high SPARC expression was shown to be a negative prognostic biomarker in pancreatic cancer. This assumption was namely based on a study including 299 patients with resected pancreatic cancer that described high stromal SPARC expression to be associated with a worse patient outcome (Infante et al, 2007). 
Table 2. Univariable and multivariable proportional hazards regression for prediction of DFS and OS

\begin{tabular}{|c|c|c|c|}
\hline $\begin{array}{l}\text { DFS } \\
\text { univariable } \\
\mathrm{HR} 95 \% \mathrm{Cl}\end{array}$ & $\begin{array}{c}\text { DFS } \\
\text { multivariable } \\
\text { HR } 95 \% \mathrm{Cl}\end{array}$ & $\begin{array}{c}\text { OS } \\
\text { univariable } \\
\mathrm{HR} 95 \% \mathrm{Cl}\end{array}$ & $\begin{array}{c}\text { OS } \\
\text { Multivariable } \\
\text { HR } 95 \% \mathrm{Cl}\end{array}$ \\
\hline \multicolumn{4}{|c|}{ Stromal density } \\
\hline \multicolumn{4}{|c|}{ Moderate vs loose } \\
\hline 1.74 & 2.66 & 2.09 & 2.68 \\
\hline $0.88-3.41$ & $1.30-5.50$ & $1.04-4.21$ & $1.28-5.60$ \\
\hline$P=0.11$ & $P=0.008$ & $P=0.039$ & $P=0.009$ \\
\hline \multicolumn{4}{|c|}{ Dense vs moderate } \\
\hline 2.78 & 3.52 & 3.06 & 3.42 \\
\hline $1.48-5.22$ & $1.81-6.80$ & $1.59-5.91$ & $1.74-6.73$ \\
\hline$P=0.002$ & $P<0.001$ & $P=0.001$ & $P<0.001$ \\
\hline \multicolumn{4}{|l|}{$\alpha-S M A$} \\
\hline \multicolumn{4}{|l|}{ High vs low } \\
\hline 0.62 & 0.53 & 0.62 & 0.52 \\
\hline $0.39-1.00$ & $0.31-0.88$ & $0.38-0.99$ & $0.31-0.89$ \\
\hline$P=0.05$ & $P=0.015$ & $P=0.05$ & $P=0.018$ \\
\hline \multicolumn{4}{|c|}{ Treatment Arm } \\
\hline \multicolumn{4}{|l|}{ Obs vs Gem } \\
\hline 0.59 & 0.49 & 0.82 & 0.78 \\
\hline $0.42-0.82$ & $0.34-0.71$ & $0.58-1.14$ & $0.55-1.12$ \\
\hline$P=0.002$ & $P<0.001$ & $P=0.23$ & $P=0.183$ \\
\hline
\end{tabular}

\begin{tabular}{|c|c|c|c|}
\hline \multicolumn{4}{|l|}{ T stage } \\
\hline \multicolumn{4}{|c|}{ pT3-4 vs pT1-2 } \\
\hline 0.60 & 0.40 & 0.59 & 0.40 \\
\hline $0.33-1.12$ & $0.21-0.77$ & $0.32-1.1$ & $0.21-0.76$ \\
\hline$P=0.10$ & $P=0.006$ & $P=0.090$ & $P=0.005$ \\
\hline
\end{tabular}

\begin{tabular}{|c|c|c|c|}
\hline \multicolumn{4}{|l|}{ pN1 vs pN0 } \\
\hline 0.68 & 0.68 & 0.72 & 0.71 \\
\hline $0.44-1.0$ & $0.44-1.05$ & $0.48-1.07$ & $0.47-1.08$ \\
\hline$P=0.052$ & $P=0.084$ & $P=0.10$ & $P=0.11$ \\
\hline
\end{tabular}

\begin{tabular}{|c|c|c|c|}
\hline \multicolumn{4}{|l|}{ Grading } \\
\hline \multicolumn{4}{|l|}{ G3 vs G1-2 } \\
\hline 0.55 & 0.56 & 0.56 & 0.54 \\
\hline $0.39-0.78$ & $0.39-0.80$ & $0.40-0.78$ & $0.38-0.77$ \\
\hline$P=0.001$ & $P=0.001$ & $P=0.001$ & $P=0.001$ \\
\hline \multicolumn{4}{|c|}{ Resection margin } \\
\hline \multicolumn{4}{|l|}{ R1 vs R0 } \\
\hline 0.92 & & 0.99 & \\
\hline $0.60-1.42$ & & $0.65-1.51$ & \\
\hline$P=0.71$ & & $P=0.97$ & \\
\hline
\end{tabular}

Our data suggest that a dense stroma cannot easily be equalised with high SPARC expression, as a dense stroma reaction was associated with a significantly increased survival in our study population and no correlation could be found between stromal density and SPARC expression in the here-presented analysis.

Our study investigated stroma quality and $\alpha$-SMA expression in a well-characterised study population group with concrete followup data concerning DFS and OS. The CONKO-001 study compared in a prospective randomised phase III trial adjuvant gemcitabine to observation only and established gemcitabine as adjuvant standard treatment in resected pancreatic cancer (Oettle et al, 2013). The study provides data for patients treated with adjuvant gemcitabine as well as for untreated patients, so the
CONKO-001 study is considered to be a unique and valuable resource for biomarker research.

For our here-presented analysis, 183 tissue samples from of the initial 354 CONKO-001 patients were available and 162 samples were suitable for construction of TMAs. Even if the fact that only about half of tumour samples were collected must be named as a limitation of our investigations, the data regarding clinical and histopathological features of the subset are comparable with the overall intention to treat population of CONKO-001. However, the sample size must be considered as a limiting factor for statistical analysis, as for example $\mathrm{pT}$ and $\mathrm{pN}$ stages were not significantly related to survival indicating a lack of power in the study. A correlation with the lymph node ratio-as an alternative to $\mathrm{pN}$ staging-would be another interesting aspect, but these data were not available for this study. Another limitation may be the TMA approach that can only partly asses tissue-and tumourheterogeneity. On the other hand, the easy-to-apply scoring system based on $\alpha$-SMA staining can be considered as a strength of our study, as simple scoring systems might reduce the interobserver variability of immunohistochemistry.

Our study underlines the important role of $\alpha$-SMA expression in pancreatic cancer. As $\alpha$-SMA expression was not significantly linked to the 'classical' histopathological characteristics (pT stage, $\mathrm{pN}$ stage, tumour grading) of our study population, it should be supposed to give additional and more specific information about the peritumoural stroma reaction and the role of activated pancreatic stellate cells. As a new finding, the study demonstrates that patients with a dense peritumoural stroma reaction had an impressively increased DFS and OS compared with those patients with a moderate or loose stroma quality. This prognostic impact of a dense peritumoural stroma was restricted to the observation group-the patient's group that can provide data for the natural course of disease after resection and before recurrence. Mechanism of immuno-escape may be an explanation of this phenomenon as formerly supposed and the deposition of a dense stroma should possibly be understood as a physiologic effort to confine cancer activity (Erkan et al, 2008). Activation of PSC seems to precede stromal activation and this may be an explanation for the correlation of $\alpha$-SMA expression and stromal density. It remains unclear which tumours can deposit in a second step a dense stroma as a kind of capsule around resulting in a better prognosis like in colorectal cancer (Lunevicius et al, 2001) and why this effect is hampered by adjuvant gemcitabine.

Our results may be limited by the sample size, but can underline in either case that analyzing and interpreting the peritumoural stroma in pancreatic cancer remains a difficult challenge with surprising results. Our findings suggest that a dense peritumoural stroma is not associated with outcome in gemcitabine-treated patients. This might imply that a dense stroma does not necessarily corrupt response to chemotherapy.

Stroma-directed treatment may be a new and promising treatment strategy, but it remains unclear which stroma markers can help to identify patients with a potential therapeutic benefit and stroma density seems not to be a helpful marker in this context.

The prognostic role of $\alpha$-SMA expression-as a relevant biomarker with a good evidence for the activity of pancreatic stellate cells-should further be evaluated in clinical trials and may serve in the future for a more individualised therapeutic approach in pancreatic cancer patients after resection.

\section{ACKNOWLEDGEMENTS}

CONKO-001 was supported in part by a grant from Lilly Deutschland, Bad Homburg, Germany. CONKO-001 was an 
investigator-initiated trial, Lilly Deutschland had no part in the design and conduct of the trial or in the collection, analysis and interpretation of the data. Trial registration: ISRCTN34802808. No grant was necessary for the here-presented data.

\section{CONFLICT OF INTEREST}

The authors declare no conflict of interest.

\section{REFERENCES}

DeJesus-Acosta A, O'Dwyer P, Ramanathan D (2014) A phase II study of vismodegib, a hedgehog pathway inhibitor, combined with gemcitabine and nab-paclitaxel in patients with untreated pancreatic ductal adenocarcinoma. J Clin Oncol 2014(suppl 3): abstr 257.

Delany AM (2010) Matricellular proteins osteopontin and osteonectin/SPARC in pancreatic carcinoma. Cancer Biol Ther 10: 65-67.

Erkan M, Adler G, Apte MV, Bachem MG, Buchholz M, Detlefsen S, Esposito I, Friess H, Gress TM, Habisch H-J, Hwang RF, Jaster R, Kleeff J, Klöppel G, Kordes C, Logsdon CD, Masamune A, Michalski CW, Oh J, Phillips PA, Pinzani M, Reiser-Erkan C, Tsukamoto H, Wilson J (2012a) StellaTUM: current consensus and discussion on pancreatic stellate cell research. Gut 61: 172-178.

Erkan M, Hausmann S, Michalski CW, Fingerle AA, Dobritz M, Kleeff J, Friess $\mathrm{H}(2012 \mathrm{~b})$ The role of stroma in pancreatic cancer: diagnostic and therapeutic implications. Nat Rev Gastroenterol Hepatol 9: 454-467.

Erkan M, Michalski CW, Rieder S, Reiser-Erkan C, Abiatari I, Kolb A, Giese NA, Esposito I, Friess H, Kleeff J (2008) The activated stroma index is a novel and independent prognostic marker in pancreatic ductal adenocarcinoma. Clin Gastroenterol Hepatol 6: 1155-1161.

Feig C, Gopinathan A, Neesse A, Chan DS, Cook N, Tuveson DA (2012) The pancreas cancer microenvironment. Clin Cancer Res 18: 4266-4276.

Fujita H, Ohuchida K, Mizumoto K, Nakata K, Yu J, Kayashima T, Cui L, Manabe T, Ohtsuka T, Tanaka M (2010) alpha-Smooth muscle actin expressing stroma promotes an aggressive tumor biology in pancreatic ductal adenocarcinoma. Pancreas 39(8): 1254-1262.

Garber K (2010) Stromal depletion goes on trial in pancreatic cancer. J Natl Cancer Inst 102: 448-450.

Hamada S, Masamune A, Shimosegawa T (2013) Novel therapeutic strategies targeting tumor-stromal interactions in pancreatic cancer. Front Physiol 4: 331.

Von Hoff DD, Ervin T, Arena FP, Chiorean EG, Infante J, Moore M, Seay T, Tjulandin SA, Ma WW, Saleh MN, Harris M, Reni M, Dowden S, Laheru D, Bahary N, Ramanathan RK, Tabernero J, Hidalgo M, Goldstein D, Van Cutsem E, Wei X, Iglesias J, Renschler MF (2013) Increased survival in pancreatic cancer with nab-paclitaxel plus gemcitabine. N Engl J Med 369: 1691-1703.

Von Hoff DD, Ramanathan RK, Borad MJ, Laheru DA, Smith LS, Wood TE, Korn RL, Desai N, Trieu V, Iglesias JL, Zhang H, Soon-Shiong P, Shi T, Rajeshkumar NV, Maitra A, Hidalgo M (2011) Gemcitabine plus nab-paclitaxel is an active regimen in patients with advanced pancreatic cancer: a phase I/II trial. J Clin Oncol 29: 4548-4554.

Infante JR, Matsubayashi H, Sato N, Tonascia J, Klein AP, Riall TA, Yeo C, Iacobuzio-Donahue C, Goggins M (2007) Peritumoral fibroblast SPARC expression and patient outcome with resectable pancreatic adenocarcinoma. J Clin Oncol 25: 319-325.

Lunardi S, Muschel RJ, Brunner TB (2014) The stromal compartments in pancreatic cancer: are there any therapeutic targets? Cancer Lett 343: $147-155$.

Lunevicius R, Nakanishi H, Ito S, Kozaki K, Kato T, Tatematsu M, Yasui K (2001) Clinicopathological significance of fibrotic capsule formation around liver metastasis from colorectal cancer. J Cancer Res Clin Oncol 127: 193-199.

Oettle H, Neuhaus P, Hochhaus A, Hartmann JT, Gellert K, Ridwelski K, Niedergethmann M, Zülke C, Fahlke J, Arning MB, Sinn M, Hinke A, Riess H (2013) Adjuvant chemotherapy with gemcitabine and long-term outcomes among patients with resected pancreatic cancer: the CONKO-001 randomized trial. JAMA 310: 1473-1481.

Oettle H, Post S, Neuhaus P, Gellert K, Langrehr J, Ridwelski K, Schramm H, Fahlke J, Zuelke C, Burkart C, Gutberlet K, Kettner E, Schmalenberg H, Weigang-Koehler K, Bechstein W-O, Niedergethmann M, Schmidt-Wolf I, Roll L, Doerken B, Riess H (2007) Adjuvant chemotherapy with gemcitabine vs observation in patients undergoing curative-intent resection of pancreatic cancer: a randomized controlled trial. JAMA 297: 267-277.

Ramírez N, Viúdez A, Hernández-García I, Guerrero D, Gómez-Dorronsoro M, Herrera FJ, Vila J, Beloki L, Ciaúrriz M, Mansilla C, Vera R (2014) Stellate cells, a point of light in the dark night of pancreatic cancer. Br J Cancer 111: 1676-1677.

Seufferlein T, Porzner M, Becker T, Budach V, Ceyhan G, Esposito I, Fietkau R, Follmann M, Friess H, Galle P, Geißler M, Glanemann M, Gress T, Heinemann V, Hohenberger W, Hopt U, Izbicki J, Klar E, Kleeff J, Kopp I, Kullmann F, Langer T, Langrehr J, Lerch M, Löhr M, Lüttges J, Lutz M, Mayerle J, Michl P, Möller P, Molls M, Münter M, Nothacker M, Oettle H, Post S, Reinacher-Schick A, Röcken C, Roeb E, Saeger H, Schmid R, Schmiegel W, Schoenberg M, Siveke J, Stuschke M, Tannapfel A, Uhl W, Unverzagt S, van Oorschot B, Vashist Y, Werner J, Yekebas E (2013) [S3-guideline exocrine pancreatic cancer]. Z Für Gastroenterol 51: 1395-1440.

Sinn M, Sinn BV, Striefler JK, Lindner JL, Stieler JM, Lohneis P, Bischoff S, Bläker H, Pelzer U, Bahra M, Dietel M, Dörken B, Oettle H, Riess H, Denkert C (2014) SPARC expression in resected pancreatic cancer patients treated with gemcitabine: results from the CONKO-001 study. Ann Oncol 25: 1025-1032.

Vonlaufen A, Phillips PA, Xu Z, Goldstein D, Pirola RC, Wilson JS, Apte MV (2008) Pancreatic stellate cells and pancreatic cancer cells: an unholy alliance. Cancer Res 68: 7707-7710.

Wilson JS, Pirola RC, Apte MV (2014) Stars and stripes in pancreatic cancer: role of stellate cells and stroma in cancer progression. Front Physiol 5: 52.

Yamao J, Toyokawa H, Kim S, Yamaki S, Satoi S, Yanagimoto H, Yamamoto T, Hirooka S, Matsui Y, Kwon A-H (2013) Activation of alpha-smooth muscle actin-positive myofibroblast-like cells after chemotherapy with gemcitabine in a rat orthotopic pancreatic cancer model. J Hepato Biliary Pancreat Sci 20: 206-213.

This work is published under the standard license to publish agreement. After 12 months the work will become freely available and the license terms will switch to a Creative Commons AttributionNonCommercial-Share Alike 3.0 Unported License. 